\title{
Effects of an Agro-Healing Activity Program on the Physiological Condition of Adults with Chronic Metabolic Diseases
}

\author{
Hye Sook Jang ${ }^{1}$, Eunha Yoo ${ }^{1}$, Sun-Jin Jeong ${ }^{1 *}$, Jae Soon Kim ${ }^{1}$, and Doo Young Ryu ${ }^{2}$ \\ ${ }^{1}$ National Institute of Horticultural \& Herbal Science, RDA, Wanju-gun 55365, Korea \\ ${ }^{2}$ Agricultural Technology Center, Sunchang-gun, Jeollabuk-do 56025, Korea
}

\section{ABSTRACT}

This study was conducted to find out the effects of agro-healing activities and strolling on the physiological conditions of patients with chronic metabolic diseases. A total of 11 subjects participated in agro-healing activities, and their average age was $49.6 \pm 11.3$. Changes in their salivary cortisol were compared and analyzed before and after participating in the agro-healing activity program, and it was found that the stress hormone decreased after participating in the program. Differences in brain wave between before and after participating in agro-healing activities and strolling were measured, and the ratio of alpha to high beta (RAHB) of subjects increased in most of the measured points after participating in agro-healing activities. In addition, the increase in the value in the temporal lobe (T3) that controls mortor skills and occipital lobe (O2) that controls visual functions indicates that the activities were effective in improving relaxation and stability. The relative low beta (RLB) power spectrum of subjects also statistically significantly decreased in the right occipital lobe (O2) that controls visual functions, the relative mid beta (RMB) and relative high beta (RHB) power spectrum decreased in the left temporal lobe (T3) and the right occipital lobe (O2). These results indicate that the activities were effective in improving relaxation under no stress and tension. The systolic and diastolic blood pressure of subjects after participating in agro-healing activities decreased from the prehypertension level to the normal level. These results indicate that horticultural activities and visual elements in healing farms, such as agro-healing activities and strolling, have positive impacts on patients with chronic metabolic diseases accompanied with diabetes or high blood pressure by increasing the brain activity and psycho-physiological conditions of participants.

Keywords: concentration, electrocardiogram, electroencephalogram, psychology, relaxation, stability

\section{Introduction}

Chronic metabolic diseases are diseases that are caused by imbalances in carbohydrates, lipid, proteins, vitamines, minerals and body fluids (Chronic metabolic disease, n.d.) and the main causes of the imbalances are unhealthy lifestyles such as obesity, lack of exercise and overnutrition (Metabolic syndrome, n.d.). These diseases can be characterized by symptoms lasting over three months and pathological conditions that are difficult to recover, and also re- quire long-term treatments and observations due to complications (Oh et al., 2001). Chronic metabolic diseases include high blood pressure, cardio and cerebrovascular diseases, diabetes, liver diseases, cancers, hyperlipidemia and arthritis, and out of them, high blood pressure and diabetes are two of the most common chronic diseases (In, 2013). In addition, as they are very closely related to cardio and cerebrovascular diseases that top the list of causes of death in Korea, it is important to prevent and manage high blood pressure from the perspective of national health promotion.

This study was supported by the 2019 Horticultural and Herbal Science Program of the National Institute of Horticultural and Herbal Science of the Rural Development Administration (PJ01137604).

Received: July 10, 2019, Revised: July 17, 2019, Accepted: July 30, 2019

First author: Hye Sook Jang, jhs915@korea.kr, (1) https://orcid.org/0000-0003-3011-8694

*Corresponding author: Sun-Jin Jeong, sunjin75@korea.kr, (1) https://orcid.org/0000-0001-6279-9733 
High blood pressure is defined as systolic blood pressure of $140 \mathrm{mmHg}$ or higher, and diastolic blood pressure of $90 \mathrm{mmHg}$ or higher, and when systolic and diastolic pressure is lower than $120 \mathrm{mmHg}$ and $80 \mathrm{mmHg}$, it is recognized as normal blood pressure (Korean Society of Hypertension [KSH], 2013). In particular, high blood pressure is one of the most commonly diagnosed chronic diseases among those in their 30s or older in Korea with the prevalence rate of over 30\% (KSH, 2013). It has already been known that proper selection of drugs and changing lifestyles are essential to effectively manage patients with diabetes accompanied by high blood pressure, and prevent complications (Evert et al., 2013). Unhealthy lifestyles such as lack of physical activity, overnutrition, irregular meals, stress, smoking and drinking have continued to increase the risks of chronic diseases (Korea Centers for Disease Control and Prevention, 2015), which has led to other issues such as increasing death rates and medical expenses, and reducing physical functions and quality of life (Roitman and La Fontaine, 2011). Chronic diseases such as high blood pressure and diabetes need to be prevented rather then focusing on treatments after occurrence, and the most effective way is properly combining medicinal and dietary therapies and exercises (So et al, 2006). Exercises such as walking and strolling are helpful for patients with high blood pressure such as lowering blood pressure, improve cardio pulmonary functions and reducing weight and stress (KSH, 2013), and they also eliminate stress sources, improve a sense of stability or happiness in the body and mind and increase the level of serotonin (Allison and Keller, 1997).

In particular, since long lasting stress affects the central and peripheral nervous systems, it has been recognized as a main cause of chronic diseases (Fuchs and Flögge, 1995; Segerstrom and Miller, 2004). Long-lasting stress also causes emotional anxiety and conflicts and causes continuous tension on the autonomic nervous system including the sympathetic and parasympathetic nervous systems, as well as mental and physical disorders and diseases (McKenzie-Mohr et al., 1995). On the other hand, once stress is reduced, positive emotions replace and suppress negative ones, reducing the activity of the sympathetic nervous system (Ulrich, 1986). In addition, physical activities in nature are known to have positive effects on psychological conditions such as improv- ing self-esteem and a change of mood compared to those in artificial environments (Yamaguchi et al., 2006). Shin et al. (2007) conducted a forest healing camp for three days and two nights for subjects who had depression for a certain period of time, and reported that activities in forests, experiences that utilize nature as well as agro-healing activities were effective in improving the physiological stability of humans.

The demand for agro-healing programs was standardized depending on the characteristics of individuals such as age, gender, academic background and vocation, whether they experience diseases and their type (Park et al., 2012), but there are not many agro-healing programs. In particular, the most important thing in developing agro-healing programs for certain targets such as patients with metabolic diseases is to identify elements that are required to develop customized agro-healing programs (Lee et al., 2011). From this perspective, it is urgently needed to develop and standardize customized programs in order to maximize healing effects (Park et al., 2013). Therefore, this study aimed to verify the effects of agro-healing programs that can reduce the risks of chronic metabolic diseases and stress, and that are easily accessible by even those who do not have any experience, and also to provide base information for developing and executing various effective agro-healing programs depending on the characteristics of targets through follow-up studies.

\section{Research Methods}

\section{Selection of subjects}

To identify the effects of agro-healing activities and strolling on the physiological conditions of patients with chronic metabolic diseases, and the following physiological conditions of a total of 11 male and female participants of which average age was $49.6 \pm 11.3$ were measured and analyzed: pre and post-activity electroencephalogram (EEG), electrocardiogram (ECG), blood pressure and salivary cortisol (saliva collection system). Subjects were recruited through online and offline bulletin boards of the Sunchang Agricultural Technology Center according to the criteria 
for the selection of subjects in general brain studies (Jang et al., 2017; Lee et al., 2009; Son et al., 1998, 1999). The final subjects were selected after conducting and analyzing the results of screening tests on the recruited subjects to ensure the selected subjects were suitable for this test as a patient with chronic metabolic disease. The selected subjects were fully informed of the purpose of this study, the content of agro-healing activities, and measurement items and methods, and signed a consent form for voluntary participation. Subjects were instructed not to drink alcohol for two days before the experiment, and not to drink any beverages that might stimulate blood pressure and saliva such as coffee, black tea and green tea for two hours before measurement. Since the number of subjects who had chronic diseases and an access to healing farms where agro-healing programs was not high enough to generalize the results of this study, it will be necessary to continue to research this.

\section{Laboratory environmental conditions}

The physiological conditions of subjects including EEG, ECG and blood pressure were measured in a healing farm in Sunchang (Fig. 1). As the outdoor temperature was too low, their physiological conditions were measured in an indoor space of the healing farm while subjects were looking at the outdoor view of the healing farm. The size of the space was length $7.0 \mathrm{~m}$, width $6.0 \mathrm{~m}$, and height $2.6 \mathrm{~m}$, and the mean intensity of illumination was $700 \pm 10$ lux (UT383 Mini Light Meters, Japan). The mean moisture and temperature were $70 \pm 10 \%$ (TR-72Ui, T\&D CORP., Japan), and $24 \pm 0.5^{\circ} \mathrm{C}$ respectively.

\section{Materials and methods}

The program used in this study was designed considering the environmental conditions of the healing farm and the fact that subjects were chronic disease patients, The program was composed of the following agro-healing activities: tree training and pruning in a vegetable garden within the healing farm, shaking beans for 50 minutes to be used to make healthy gangjeong, rice crackers, in the next session. Strolling around the farm for 40 minutes was also included since walking and running are effective for patients with high blood pressure and diabetes but it is recommended to walk for 30-60 minutes first before instead of walking for a long period of time from the beginning (Lee, 2018). The physiological conditions of subjects including EEG, ECG, blood pressure and saliva collection were measured before and after each agro-healing activity (Fig. 1). Prior to measuring the EEG and ECG of subjects, their blood pressure and pulse were measured using a portable digital blood pressure monitor (HEM-1000, OMRON, Japan) that uses an oscilloscope. The EEG and ECG of subjects were measured using a portable wireless EEG monitor that is easily wearable (BIOS-SW8, BioBrain Inc., Daejeon, Korea). Subjects were informed of the overall explanation of the experiment, signed a consent form and were also ensured to familiarize themselves with the environment while placing the monitors prior to measurement. Electrodes were placed according to the International 10-20 System of electrode placement (Berger, 1929), A dry cap equipped with seven channels of electrodes on the frontal lobes (F3, F4), temporal lobes (T3, T4), central zone (Cz),

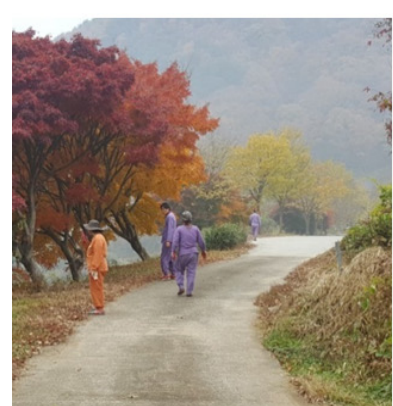

(A)

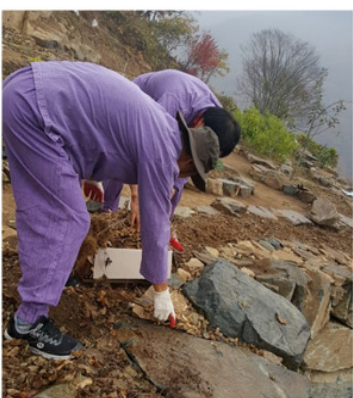

(B)

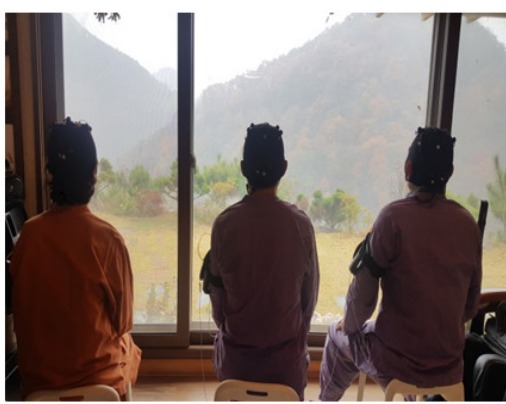

(C)

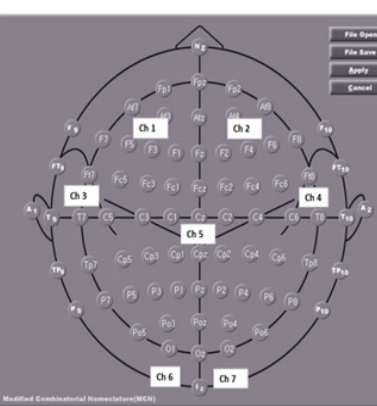

(D)

Fig. 1. Agro-healing program includes walking $(A)$ and gardening activity (B). Images of subjects wearing an EEG-cap at indoors (C) and positions of 7 electrodes on the top view of the scalp (D). 
and occipital lobes $(\mathrm{O} 1, \mathrm{O} 2)$ was used. A reference electrode was placed behind the ear, and for measuring the ECG of subjects a disposable electrode was placed on the inner part of the left and right wrists. A total of 10 electrodes were placed (Fig. 1). Earplugs were used to ensure that subjects were not exposed to extraneous noise. Subjects were ensured to relax for two minutes prior to measurement.

The saliva of subjects was collected by instructing them to spit. There are many methods to collect saliva to measure salivary cortisol, but the way of collecting saliva by instructing subjects to directly spitting or drooling into a certain container is the easiest and simplest (Zava, 2004). However, it is difficult to collect a sufficient amount of quality saliva, and the saliva collected using the method can be easily contaminated by hand or air contacts (Gordon et al., 2005). For this reason, saliva was carefully collected.

\section{Statistical analysis}

Statistical analysis was conducted using IBM SPSS ver. 25.0. and a Wilcoxon paired signed rank test was performed on the measured results of EEG, ECG, blood pressure and saliva. The data of physiological signals were analyzed by calculating the power spectrum of frequency bands using the algorithm of fast fourier transformation (FFT). The generated data of each electrode were standardized as a mean value, and based on the results the mean value of each electrode was calculated and the brain maps were written and reviewed. Variations in electric energy were marked in the brain maps using different colors as follows: low electrical potential in blue and high electrical potential in red (Fig. 2).

\section{Results and Discussion}

\section{Changes in the EEG of participants in agro-healing activities and strolling}

Changes in the EEG of participants in agro-healing activities and strolling were analyzed, and the results showed that the ratio of alpha to high beta (RAHB), an indicator of stability and relaxation, after participating in agro-heal- ing activities and strolling was higher in most of the measured points than before participating in the activities. The value in the temporal lobe (T3) that controls motor skills and the occipital lobe $(\mathrm{O} 2)$ that controls visual functions was statistically significantly high, which indicates the effects of stability and relaxation. The relative low beta (RLB), an indicator of attention accompanied with arousal, decreased in the right occipital lobe $(\mathrm{O} 2)$ that controls visual functions, and the relative mid beta (RMB), an indicator of attention and concentration accompanied with mild tension, and the relative high beta (RHB), an indicator of tension and stress, decreased in the left temporal lobe (T3) and the right occipital lobe $(\mathrm{O} 2)$, and the results were statistically significant, indicating that the activities were effective in improving stability and relaxation under no stress and tension. In addition, the relative slow alpha (RSA), the relative fast alpha (RFA) also increased after participating in agro-healing activities and strolling, although the results were not statistically significant (Tables 1 and 2; Figs. 1 and 2). The results can be interpreted as that activities in healing farms such as agro-healing activities and strolling and visual elements in the surrounding environments of farms increase the ratio of alpha waves and have positive impacts on the brain activity, stability and relaxation of patients with chronic metabolic diseases including diabetes and high blood pressure (Kim et al., 2002; Son et al., 1998, 1999). In addition, strolling was also reported to improve physiological functions, immunity and physical strength, and have positive impacts on psychological conditions such as reducing beta waves and cortisol, a stress hormone (Lee, 2016), which supports the results of this study that strolling around a healing farm had positive effects.

\section{Changes in the autonomic nervous system of participants in agro-healing program}

Changes in the autonomic nervous system of patients with chronic metabolic diseases after participating in agro-healing activities and strolling were analyzed. The results showed that $\mathrm{LF}$ (low frequency)/LF $+\mathrm{HF}$, an indicator of the activity of the sympathetic nervous system, decreased, and that the $\mathrm{HF}$ (high frequency)/LF $+\mathrm{HF}$, an indicator of the activity of the parasympathetic nervous system, increased after par- 
Before

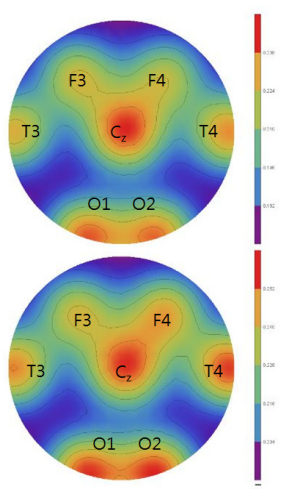

RSA

Before

After

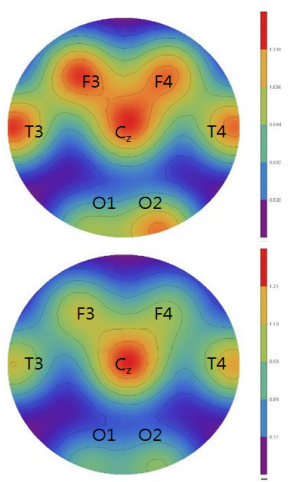

RSMT

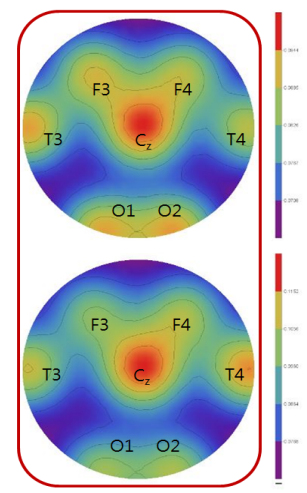

$\operatorname{RFA}(p<.05)$

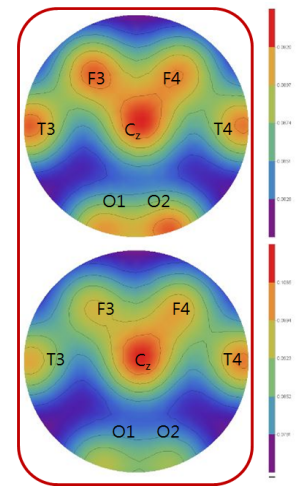

$\operatorname{RLB}(p<.05)$

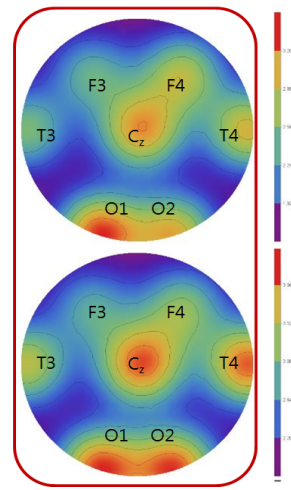

$\operatorname{RAHB}(p<.05)$

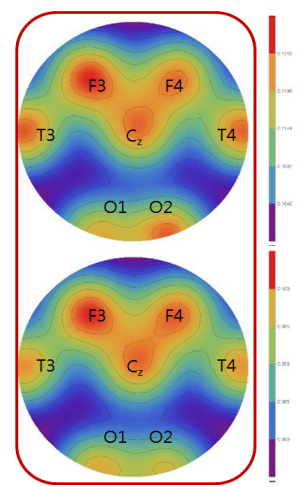

$\operatorname{RMB}(p<.05)$

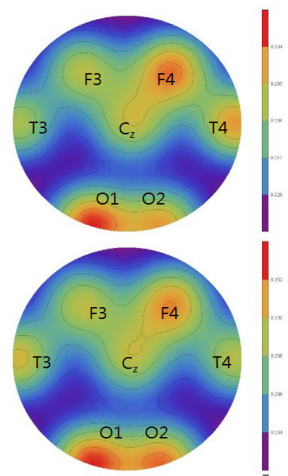

RT

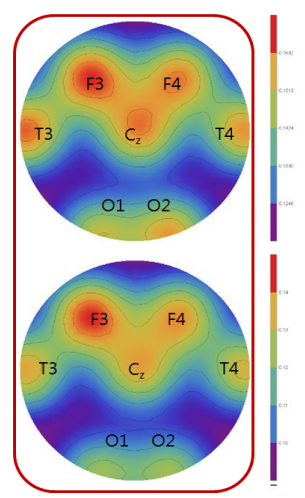

$\mathrm{RHB}(p<.05)$

Fig. 2. Electroencephalographic (EEG) mapping for change of brain wave of total participants before and after agro-healing activity program. Blue and red indicate low and high electrical potential, respectively. RSA=relative slow alpha power spectrum; RFA=relative fast alpha power spectrum; RAHB=ratio of alpha to high beta; RT=relative theta power spectrum; $\mathrm{RSMT}=$ ratio of (SMR-Mid Beta) to theta; $R L B=$ relative low-beta power spectrum; RMB=relative mid- beta power spectrum; $\mathrm{RHB}=$ relative high-beta power spectrum; $\mathrm{F}=$ frontal lobes; $\mathrm{T}=$ temporal lobe; $\mathrm{Cz}=$ =central zone; $\mathrm{O}=$ =ccipital lobes; odd and even numbers indicate left and right hemispheres, respectively.

Table 1. Significance on the differences of brain wave changes before and after agro-healing activity program

\begin{tabular}{cccccccl}
\hline EEG & F3 & F4 & \multicolumn{1}{c}{ T3 } & T4 & Cz & O1 & \multicolumn{1}{c}{ O2 } \\
\hline RSA & $-0.533^{\mathrm{z}}$ & -1.423 & -1.511 & -1.511 & -0.089 & -0.445 & -1.067 \\
RFA & -0.889 & 0.000 & -0.089 & -0.978 & 0.000 & -0.533 & -1.511 \\
RAHB & -0.800 & -0.889 & $-2.845^{* *}$ & -1.600 & -1.511 & -0.622 & $-2.490^{*}$ \\
RT & -0.622 & -0.800 & -0.978 & -0.178 & -0.445 & -0.622 & -0.978 \\
RSMT & -1.067 & -0.889 & -0.889 & -0.089 & -0.445 & -0.622 & -1.511 \\
RLB & -1.245 & -0.178 & -0.533 & -0.178 & -0.267 & -0.622 & $-2.401^{*}$ \\
RMB & -1.511 & -1.156 & $-2.223^{*}$ & -1.778 & -1.423 & -0.800 & $-2.845^{* *}$ \\
RHB & -0.889 & -1.245 & $-2.401^{*}$ & -1.423 & $-1.956^{*}$ & -0.711 & $-2.490^{*}$ \\
\hline
\end{tabular}

Note. $\mathrm{EEG}=$ electroencephalogram; $\mathrm{RSA}=$ relative slow alpha power spectrum; $\mathrm{RFA}=$ relative fast alpha power spectrum; $\mathrm{RAHB}=$ ratio of alpha to high beta; RT=relative theta power spectrum; RSMT=ratio of (SMR-Mid Beta) to theta; RLB=relative low-beta power spectrum; $\mathrm{RMB}=$ relative mid- beta power spectrum; $\mathrm{RHB}=$ relative high-beta power spectrum; $\mathrm{F}=$ frontal lobes; $\mathrm{T}=$ temporal lobe; $\mathrm{Cz}=\mathrm{central} z o n e ;$ $\mathrm{O}=$ occipital lobes; odd and even numbers indicate left and right hemispheres, respectively.

${ }^{\mathrm{z}} \mathrm{Z}$ value.

${ }^{*} p<.05,{ }^{*} p<.01$ by Wilcoxon paired signed-rank test $(\mathrm{n}=11)$. 
Table 2. Brain wave of participants before and after agro-healing activity program

\begin{tabular}{|c|c|c|c|c|c|c|c|c|}
\hline EEG & Treatment & F3 & F4 & $\mathrm{T} 3$ & $\mathrm{~T} 4$ & $\mathrm{Cz}$ & $\mathrm{O} 1$ & $\mathrm{O} 2$ \\
\hline \multirow{2}{*}{ RSA } & Before & $0.181 \pm 0.02$ & $0.166 \pm 0.02$ & $0.198 \pm 0.02$ & $0.221 \pm 0.03$ & $0.251 \pm 0.04$ & $0.237 \pm 0.02$ & $0.229 \pm 0.02$ \\
\hline & After & $0.188 \pm 0.03$ & $0.206 \pm 0.03$ & $0.231 \pm 0.04$ & $0.262 \pm 0.04$ & $0.253 \pm 0.04$ & $0.258 \pm 0.04$ & $0.263 \pm 0.04$ \\
\hline \multirow{2}{*}{ RFA } & Before & $0.073 \pm 0.01$ & $0.066 \pm 0.01$ & $0.084 \pm 0.01$ & $0.069 \pm 0.01$ & $0.102 \pm 0.02$ & $0.081 \pm 0.01$ & $0.083 \pm 0.01$ \\
\hline & After & $0.070 \pm 0.01$ & $0.079 \pm 0.02$ & $0.093 \pm 0.02$ & $0.112 \pm 0.03$ & $0.128 \pm 0.04$ & $0.076 \pm 0.01$ & $0.081 \pm 0.02$ \\
\hline \multirow{2}{*}{ RAHB } & Before & $1.582 \pm 0.19$ & $2.292 \pm 0.60$ & $1.827 \pm 0.20$ & $2.647 \pm 0.61$ & $2.933 \pm 0.55$ & $3.554 \pm 0.62$ & $2.727 \pm 0.45$ \\
\hline & After & $1.850 \pm 0.26$ & $2.518 \pm 0.33$ & $3.074 \pm 0.45$ & $4.290 \pm 1.18$ & $4.260 \pm 1.17$ & $4.518 \pm 0.66$ & $4.428 \pm 0.66$ \\
\hline \multirow{2}{*}{ RT } & Before & $0.211 \pm 0.02$ & $0.284 \pm 0.06$ & $0.205 \pm 0.02$ & $0.267 \pm 0.05$ & $0.211 \pm 0.02$ & $0.320 \pm 0.05$ & $0.253 \pm 0.04$ \\
\hline & After & $0.247 \pm 0.03$ & $0.315 \pm 0.04$ & $0.278 \pm 0.05$ & $0.250 \pm 0.04$ & $0.237 \pm 0.05$ & $0.371 \pm 0.06$ & $0.333 \pm 0.07$ \\
\hline \multirow{2}{*}{ RSMT } & Before & $1.193 \pm 0.23$ & $1.072 \pm 0.26$ & $1.228 \pm 0.23$ & $1.135 \pm 0.25$ & $1.163 \pm 0.24$ & $0.733 \pm 0.13$ & $1.094 \pm 0.25$ \\
\hline & After & $0.878 \pm 0.11$ & $0.724 \pm 0.14$ & $0.950 \pm 0.19$ & $1.092 \pm 0.26$ & $1.336 \pm 0.40$ & $0.637 \pm 0.15$ & $0.743 \pm 0.18$ \\
\hline \multirow{2}{*}{ RLB } & Before & $0.090 \pm 0.01$ & $0.081 \pm 0.01$ & $0.093 \pm 0.01$ & $0.089 \pm 0.01$ & $0.094 \pm 0.01$ & $0.080 \pm 0.00$ & $0.091 \pm 0.01$ \\
\hline & After & $0.080 \pm 0.01$ & $0.085 \pm 0.01$ & $0.091 \pm 0.01$ & $0.102 \pm 0.02$ & $0.110 \pm 0.03$ & $0.074 \pm 0.01$ & $0.068 \pm 0.01$ \\
\hline \multirow{2}{*}{$\mathrm{RMB}$} & Before & $0.128 \pm 0.01$ & $0.112 \pm 0.01$ & $0.121 \pm 0.01$ & $0.120 \pm 0.01$ & $0.109 \pm 0.01$ & $0.097 \pm 0.01$ & $0.118 \pm 0.01$ \\
\hline & After & $0.106 \pm 0.01$ & $0.095 \pm 0.01$ & $0.090 \pm 0.01$ & $0.089 \pm 0.01$ & $0.090 \pm 0.01$ & $0.083 \pm 0.01$ & $0.076 \pm 0.01$ \\
\hline \multirow{2}{*}{ RHB } & Before & $0.169 \pm 0.01$ & $0.144 \pm 0.02$ & $0.159 \pm 0.01$ & $0.143 \pm 0.02$ & $0.141 \pm 0.01$ & $0.115 \pm 0.02$ & $0.138 \pm 0.02$ \\
\hline & After & $0.151 \pm 0.01$ & $0.123 \pm 0.01$ & $0.120 \pm 0.01$ & $0.112 \pm 0.01$ & $0.112 \pm 0.01$ & $0.090 \pm 0.01$ & $0.092 \pm 0.01$ \\
\hline
\end{tabular}

Note. Values are mean \pm standard error $(\mathrm{n}=11)$.

$\mathrm{EEG}=$ electroencephalogram; RSA=relative slow alpha power spectrum; $\mathrm{RFA}=$ relative fast alpha power spectrum; $\mathrm{RAHB}=$ ratio of alpha to high beta; RT=relative theta power spectrum; RSMT=ratio of (SMR-Mid Beta) to theta; $R L B=$ relative low-beta power spectrum; $\mathrm{RMB}=$ relative mid- beta power spectrum; $\mathrm{RHB}=$ relative high-beta power spectrum; $\mathrm{F}=$ frontal lobes; $\mathrm{T}=$ temporal lobe; $\mathrm{Cz}=\mathrm{central} z$ zone; $\mathrm{O}=$ occipital lobes; odd and even numbers indicate left and right hemispheres, respectively.

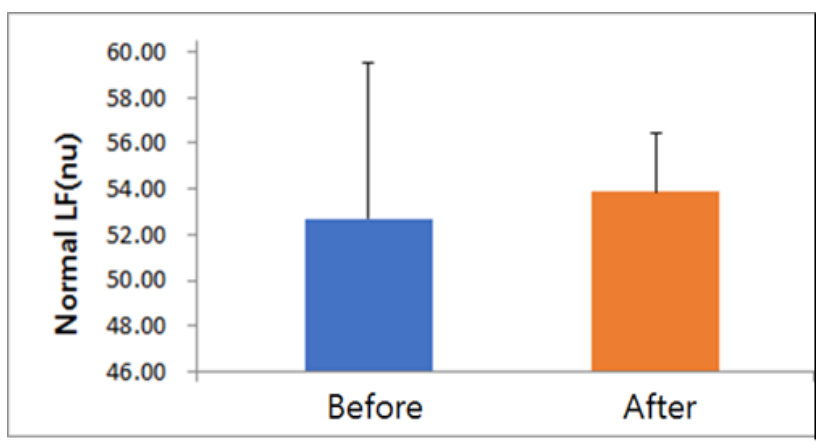

Fig. 3. Difference of sympathetic nerve between before and after agro-healing activity.

ticipating in the program, but they were not statistically significant (Figs. 3 and 4). The results indicate that the visual elements of the landscape of the healing farm and walking tend to reduce tension. Park (2010) adopted an experimental approach to forest healing effects using forest recreation activities, and reported that looking at the landscape of forests suppressed the activity of the sympathetic nervous system compared to urban environments. The re-

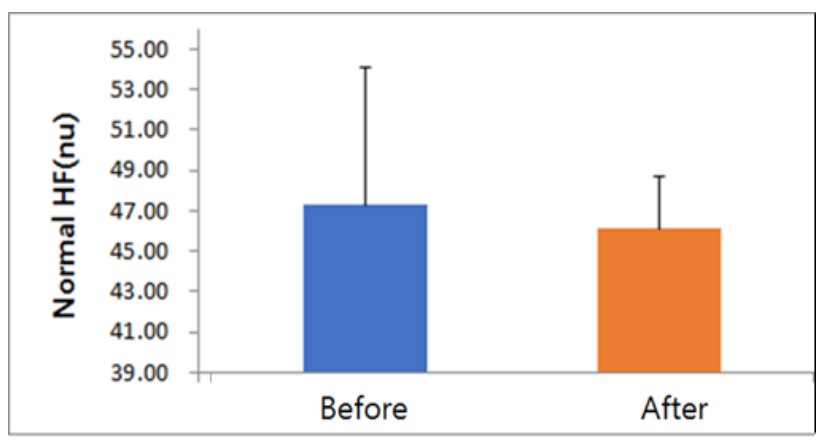

Fig. 4. Difference of parasympathetic nerve between before and after agro-healing activity.

sults seem to coincide with those of this study that the environment of the healing farm suppressed the activity of the sympathetic nervous activity.

\section{Changes in the blood pressure and pulse rate of participants in agro-healing program}

Changes in the blood pressure and pulse rate of patients with chronic metabolic diseases after participating in ago-heal- 
ing activities and strolling were analyzed. The results showed that the mean systolic blood pressure after participating in the program was $117.00 \mathrm{mmHg}$ down by $82.91 \mathrm{mmHg}$ from the mean value before participating in the program (122.91 $\mathrm{mmHg}$ ) and that the mean diastolic blood pressure after participating in the program was $77.36 \mathrm{mmHg}$ down by $5.55 \mathrm{mmHg}$ from the mean value before participating in the program $(82.91 \mathrm{mmHg})$, and the results were statistically significant. The systolic blood pressure before participating in healing activities in the healing farm such as activities in vegetable gardens and strolling decreased from the prehypertension level to the normal level after participating in the program, which indicates that agro-healing activities were effective in reducing blood pressure (Table 3). In addition, the heart rate of participants after participating in agro-healing activities and strolling tended to increase compared to the value before participating in the program, and the results were statistically significant. However, the value was within the mean pulse rate before and after participating in the program (Table 4). The results of KSH (2013) that exercises such as walking and strolling reduced the blood pressure and stress of patients with high blood pressure supported the results of this study.

\section{Changes in salivary cortisol after participating in agro-healing activities and strolling}

Changes in the level of salivary cortisol after participating in agro-healing activities and strolling were analyzed. The mean level of salivary cortisol, a stress hormone, after participating in the program was $4.373 \mu \mathrm{g} / \mathrm{dl}$, down by $3.127 \mu \mathrm{g} / \mathrm{dl}$, from $7.500 \mu \mathrm{g} / \mathrm{dl}$ before participating in the program $(z=-2.237, p<.05)$, and the differences were statistically significant (Table 5). The results indicate that agro-healing activities and strolling in healing farms reduce the level of cortisol, a stress hormone. These results were similar with the results of Jang et al. (2018) that the level of cortisol statistically significantly decreased after participating in the program of plant cultivation activities, and the results of Lee et al. (2013) that the level of depression and salivary cortisol of prisoners who were soon to be released statistically significantly decreased after participating in the program of horticultural activities. In addition, Grahn and Stigsdotter (2003) reported that strolling in natural

Table 3. Blood pressure changes in participants between before and after agro-healing activity

\begin{tabular}{|c|c|c|c|c|}
\hline \multirow{2}{*}{ Blood pressure } & \multicolumn{2}{|c|}{$\mathrm{M} \pm \mathrm{SD}$} & \multirow{2}{*}{$z$} & \multirow{2}{*}{$p$} \\
\hline & Before & After & & \\
\hline Systolic blood pressure & $122.91 \pm 12.43$ & $117.00 \pm 14.65$ & -2.854 & $.004 * *$ \\
\hline Diastolic blood pressure & $82.91 \pm 10.45$ & $77.36 \pm 10.87$ & -2.316 & $.021^{*}$ \\
\hline
\end{tabular}

${ }^{*} p<.05,{ }^{* *} p<.01$ by Wilcoxon paired signed-rank test $(\mathrm{n}=11)$.

Table 4. Difference of pulse rate in participants between before and after agro-healing activity

\begin{tabular}{|c|c|c|c|c|}
\hline \multirow{2}{*}{ Pulse rate } & \multicolumn{2}{|c|}{$\mathrm{M} \pm \mathrm{SD}$} & \multirow{2}{*}{$z$} & \multirow{2}{*}{$p$} \\
\hline & Before & After & & \\
\hline Total & $67.27 \pm 6.44$ & $71.55 \pm 8.36$ & -2.185 & $.029 *$ \\
\hline
\end{tabular}

${ }^{*} p<.05$ by Wilcoxon paired signd-rank test $(\mathrm{n}=11)$.

Table 5. Cortisol level change in participants between before and after agro-healing activity

\begin{tabular}{cccccc}
\hline \multirow{2}{*}{ Cortisol value $(\mu \mathrm{g} / \mathrm{dl})$} & $\mathrm{M} \pm \mathrm{SD}$ & \multirow{2}{*}{$z$} & $p$ \\
\cline { 2 - 5 } & Before & After & -2.092 & $.036^{*}$ \\
\hline Total & $7.500 \pm 6.28$ & $4.373 \pm 2.26$ & & \\
\hline
\end{tabular}

$* p<.05$ by Wilcoxon paired signed-rank test $(\mathrm{n}=11)$. 
environments was effective in reducing stress, and that even if a short strolling reduced the level of stress hormones. The results of this study were also supported by Kaplan (2001) and Ulrich (1984) that reported that horticultural plant activities and landscapes of horticultural plants reduced the stress of subjects, maintained tranquility and also were effective in achieving psychological healing and improving the ability to recover.

\section{Conclusion}

To measure changes in the physiological conditions of patients with chronic metabolic diseases after participating in agro-healing activities and strolling, this study measured the electroencephalogram (EEG), blood pressure and salivary cortisol (saliva collection program) of a total of 11 male and female participants (average age was 49.6 \pm 11.3 ). Their physiological conditions before and after participating in the agro-healing program were compared and analyzed, and it was found that their level of cortisol, a stress hormone, after participating in agro-healing activities and strolling statistically significantly decreased $(\mathrm{z}=-2.092, p$ $<.05)$. Changes in the EEG of participants after participating in agro-healing activities and strolling were measured, and the ratio of alpha to high beta (RAHB) after participating in agro-healing activities and strolling increased in most of the measured points than before participating in the activities. The value in the temporal lobe (T3) that controls motor skills and the occipital lobe (O2) that controls visual functions was statistically significantly high, which indicates that the activities are effective in improving stability and relaxation. The relative low beta (RLB) decreased in the right occipital lobe $(\mathrm{O} 2)$ that controls visual functions, and the relative mid beta $(\mathrm{RMB})$ and the relative high beta (RHB) decreased in the left temporal lobe (T3) and the right occipital lobe $(\mathrm{O} 2)$. The results were statistically significant, indicating that the activities were effective in improving stability and relaxation under no stress and tension. Changes in the blood pressure of participants after participating in agro-healing activities were measured. It was found that the systolic and diastolic blood pressure of participants decreased from the prehypertension level to the normal level after participating in the program, and the results were statistically significant. Participating in agro-healing activities and strolling in healing farms and visual elements in healing farms increased the ratio of alpha waves of patients with chronic metabolic diseases accompanied with diabetes or high blood pressure, which indicates that the activities were effective in improving the brain activity and mental and physiological conditions of participants. owever, since the number of participants was not high enough to generalize the results of this study, it will be necessary to continue to research the positive effects of agricultural activities or agro-healing activities such as strolling on patients with chronic metabolic diseases.

\section{References}

Allison, M. and C. Keller. 1997. Physical activity in the elderly: Benefit and intervention strategies. Nurse Pract. 22(8):53-69.

Berger, H. 1929. Über das elektrekephalogram des menschen (On the human electrocephalogram). Arch. Psychiatr. Nervenkr. [Eur. Arch. Psychiatry Neurol. Sci.] 87(1):527-570. Doi.org/10.1007/BF01797193

Chronic metabolic disease. n.d. In RDA agricultural terms dictionary. Retrieved from http://nongsaro.go.kr

Evert, A.B., J.L. Boucher, M. Cypress, S.A. Dunbar, M.J. Franz, E.J. Mayer-Davis, J.J. Neumiller, R. Nwankwo, C.L. Verdi, P. Urbanski, and W.S. Yancy Jr. 2013. Nutrition therapy recommendations for the management of adults with diabetes. Diabetes Care 36(11):3821-3842. https://doi.org/10.2337/dc13-2042

Fuchs, E. and G. Flügge. 1995. Modulation of binding sites for corticotropin-releasing hormone by chronic psychosocial stress. Psychoneuroendocrinology 20(1):33-51. https://doi.org/10.1016/0306-4530(94)E0006-u

Gordon, M.K., E. Peloso, A. Auker, and M. Dozier. 2005. Effect of flavored beverage crystals on salivary cortisol enzyme-immunoreactive assay measurements. Dev. Psychobiol. 47(2):189-195. https://doi.org/10.1002/de v.20081

Grahn, P. and U.A. Stigsdotter. 2003. Landscape planning 
and stress. Urban For. Urban Green 2(1):1-18. https://doi.org/10.1078/1618-8667-00019

In, H.K. 2013, September 6. The top 10 causes of death. KCDC. Retrieved from www.cdc.go.kr

Jang, H.S., G.M. Gim, K.J. Kim, and H.H. Jung. 2017. Change of physiology and emotion according to plant-decoration activity in workers. J. People Plants Environ. 20(5):555-566. https://doi.org/10.11628/ksppe.2017.20.5.555

Jang, H.S., G.M. Gim, S.J. Jeong, and J.S. Kim. 2018. Effects of plant cultivating activity for reducing parental stress. J. People Plants Environ. 21(3):203-211. https://doi.org/10.11628/ksppe.2018.21.3.203

Kaplan, R. 2001. The nature of the view from home: Psychological benefits. Environ. Behav. 33(4):507-542. https://doi.org/10.1177/00139160121973115

Kim, E.I., S.H. Hwang, W.S. Shin, and K.W. Ahn. 2002. Physiological effect of forest types: Focused on brain wave and pulsation. J. Korean Inst. For. Recreat. 6(2):43-48.

Korea Centers for Disease Control and Prevention. 2015. 2008-2014 Regional health statistics at a glance. Cheongju, Korea: Author. Retrieved from www.cdc.go.kr

Korean Society of Hypertension. 2013. Hypertension guidelines. Seoul, Korea: IMED.

Lee, E.D., S.J. Park, R.H. Yoo, and S.J. Hong. 2011. Analysis on the activity contents of forest healing programs in Korea. J. Korean Inst. For. Recreat. 15(2):101-109.

Lee, J.H. 2018, September 7. Walking that corrects high blood pressure and diabetes. The Korea Economic Daily. Retrieved from http://www.hankyung.com

Lee, J.H., W.S. Shin, P.S. Yeoun, and R.H. Yoo. 2009. The influence of forest scenes on psychophysiological responses. J. Korean For. Soc. 98(1):88-93.

Lee, J.S. 2016. The effect of 12-weeks forest walking on functional fitness, self-efficacy, and stress in the middle-aged women. Master's thesis, Chungbuk National University, Cheongju, Korea.

Lee, S.M., J.H. Moon, S.J. Jeong, and Y.J. Lee. 2013. Effects of horticultural therapy program focusing on cultivating activities for prisoners scheduled to be released on depression and salivary cortisol. J. Korean
Soc. People Plants Environ. 16(4):173-180. https://doi.org/10.11628/ksppe.2013.16.4.173

McKenzie-Mohr, D., L.S. Nemiroff, L. Beers, and S. Desmarais. 1995. Determinants of responsible environmental behavior. J. Soc. Issues 51(4):139-156. https://doi.org/10.1111/j.1540-4560.1995.tb01352.x

Metabolic syndrome. n.d. In Hankyoung-dotcom dictionary. Retrieved from http://dic.hankyung.com

Oh, Y.H., J.J. Oh, and Y.G. Ji. 2001. The chronic disease condition and its impact on the patient's health. Sejong, Korea: Korea Institute for Health and Social Affairs.

Park, B.H., S.K. Kim, S.M. Kim, S.B. Yoon, and M.J. Park. 2013. Studies for using type and benefits of the visitors in the natural recreation forest. J. Korean Inst. For. Recreat. 17(4):59-67.

Park, B.J. 2010. Experimental approach of therapeutic effect of forest recreation activities: Focused on viewing and walking in forest environments. Doctoral dissertation, Chungnam National University, Daejeon, Korea.

Park, S.M., J.M. Woo, W. Kim, and Y.J. Lee. 2012. Sub-populations and disorders that can be applied to forest therapy. J. Korean Inst. For. Recreat. 16(1):35-42.

Roitman, J.L. and T. LaFontaine. 2011. The exercise professional's guide to optimizing health: Strategies for preventing and reducing chronic disease. Baltimore, MD: Lippincott Williams \& Wilkins.

Segerstrom, S.C. and G.E. Miller. 2004. Psychological stress and the human immune system: A meta-analytic study of 30 years of inquiry. Psychol. Bull. 130(4):601-630. https://doi.org/10.1037/0033-2909.130.4.601

Shin, W.S., P.S. Yeoun, and J.H. Lee. 2007. The impact that a forest experience influences on a human mental state stability. J. Korean Inst. For. Recreat. 11(3):37-43.

So, J.M., M.S. Kim, J.H. Seo, Y.W. Jo, S.W. Wang, H.S. Ahn, C.H. Lee, and C.H. Chae. 2006. Understanding of excercise for health. Seoul, Korea: Konkuk University Press.

Son, K.C., J.S. Lee, and J.E. Song. 1998. Effect of visual recognition of indoor plants on changes of human brain electroencephalography. J. Korean Soc. Hortic. Sci. 39(6):858-862. 
Son, K.C., J.S. Lee, and J.E. Song. 1999. Effect of visual recognition of Ficus benjamina and its photograph on changes of human brain electroencephalography and brain blood flow. J. Korean Soc. Hortic. Sci. 40(1):134-138.

Ulrich, R.S. 1984. View through a window may influence recovery from surgery. Science 224(4647):420-421. DOI:10.1126/science.6143402

Ulrich, R.S. 1986. Human responses to vegetation and landscapes, Landscape and Urban Planing 13:29-44. DOI:10.1016/0169-2046(86)90005-8

Yamaguchi, M., M. Deguchi, and Y. Miyazaki. 2006. The effects of exercise in forest and urban environments on sympathetic nervous activity of normal young adults. J. International Medical Research 34:152-159.

Zava, D. 2004, January. Saliva hormone testing. Townsend Letter for Doctors \& Patients, 120-124. 